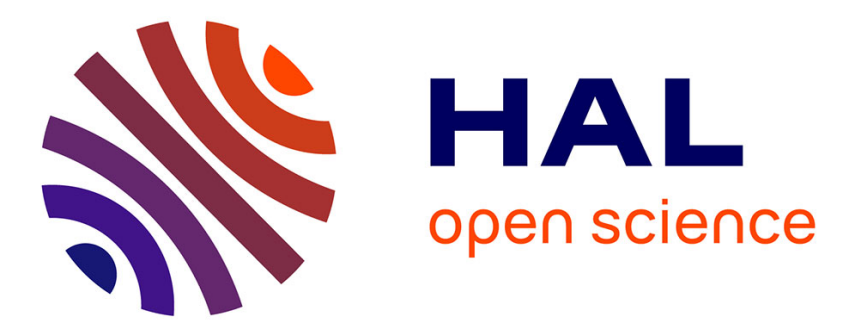

\title{
Luminescent Characteristics of Needle-Like Single Crystal Diamonds
}

Sergey Malykhin, Jonathan Houard, Rinat Ismagilov, Anton Orekhov, Angela Vella, Alexander Obraztsov

\section{- To cite this version:}

Sergey Malykhin, Jonathan Houard, Rinat Ismagilov, Anton Orekhov, Angela Vella, et al.. Luminescent Characteristics of Needle-Like Single Crystal Diamonds. physica status solidi (b), 2018, 255 (1), pp.1700189. 10.1002/pssb.201700189 . hal-01766078

\section{HAL Id: hal-01766078 \\ https://hal.science/hal-01766078}

Submitted on $20 \mathrm{Jul} 2020$

HAL is a multi-disciplinary open access archive for the deposit and dissemination of scientific research documents, whether they are published or not. The documents may come from teaching and research institutions in France or abroad, or from public or private research centers.
L'archive ouverte pluridisciplinaire HAL, est destinée au dépôt et à la diffusion de documents scientifiques de niveau recherche, publiés ou non, émanant des établissements d'enseignement et de recherche français ou étrangers, des laboratoires publics ou privés. 


\title{
Luminescent Characteristics of Needle-Like Single Crystal Diamonds
}

\author{
Sergey A. Malykhin, * Jonathan Houard, Rinat R. Ismagilov, Anton S. Orekhov, \\ Angela Vella, and Alexander N. Obraztsov
}

Luminescent properties of needle-like single crystal diamonds are investigated in a wide range of wavelength. The luminescent spectra with zero phonon lines centered at 389, 442, 468, 534, 563, 575, and $738 \mathrm{~nm}$ are detected using excitation by photons and electrons. Obtained photo- and cathodo-luminescent (PL and $C L$ ) spectra indicate presence in the singlecrystal diamond needles of nitrogen- and silicon-vacancy centers as well as substitutional or/and interstitials related to these atomic impurities. The dependencies of PL intensities of the 575 and $738 \mathrm{~nm}$ lines (related, correspondingly, to nitrogen- and silicon-vacancy centers) on excitation wavelength are determined. Time-resolved measurements are performed for the $\mathbf{5 7 5} \mathrm{nm}$ PL line. The luminescence decay behavior for this line demonstrates the presence of recombination processes with characteristic times of about $28 \mathrm{~ns}$ (on $200 \mathrm{~ns}$ time scale) and $2.8 \mathrm{~ns}$ (on $20 \mathrm{~ns}$ time scale). deposition (CVD) techniques ${ }^{[4]}$ provides expansion of industrial usage of diamond. ${ }^{[5]}$ Crystalline qualities of synthetic diamonds (which may be better than the best natural diamonds at present time ${ }^{[6]}$ ) are critical for many applications as well as properties of particular structural defects. This stimulates investigations of structural peculiarities and defects in diamond. Many of these defects attract attention because of their optical and luminescent properties. More than 500 types of the color-centers are known in diamond. ${ }^{[7]}$ Luminescent characteristics of some of them allow consideration of diamond as promising material for different optical and quantum optical applications. $^{[8,9]}$

Important advantage of CVD techniques consists in ability to produce diamond crystallites of needle-like shape ${ }^{[10,11]}$ which

\section{Introduction}

Diamond is an amazing material that combines record hardness and thermal conductivity, biocompatibility, optical transparency, and other properties attractive for numerous applications. ${ }^{[1,2]}$ Development of methods for synthesis of diamond with highpressure-high-temperature $(\mathrm{HPHT})^{[3]}$ and chemical vapor

S. A. Malykhin, Dr. R. R. Ismagilov, Prof. A. N. Obraztsov

Department of Physics

Lomonosov Moscow State University,

Leninskiye Gory 1, 119234 Moscow, Russia

E-mail: sermal92@mail.ru

S. A. Malykhin, Prof. A. N. Obraztsov

Department of Physics and Mathematics

University of Eastern Finland,

Yliopistokatu 7, 80101 Joensuu, Finland

S. A. Malykhin

Division of Solid State Physics

Lebedev Physical Institute of the Russian Academy of Sciences,

Leninskiy Prospekt 53, 119991 Moscow, Russia

J. Houard, Prof. A. Vella

Groupe de Physique des Matériaux CNRS, INSA Rouen, UNIROUEN, Normandie Univ, 76000 Rouen, France

A. S. Orekhov

National Research Center "Kurchatov Institute",

Akademika Kurchatova pl. 1, 123182 Moscow, Russia

DOI: 10.1002/pssb.201700189 may be usable for some applications. In particular diamond needles are considered for creation of optical probes and sensors. These applications require comprehensive investigation and deeper understanding of the luminescent properties of particular color-centers in this type of diamond material. In this work, we perform study of the diamond needle-like single crystals to reveal their photo- and cathode-luminescent characteristics in a wide range of wavelength, never explored before for this kind of samples. Results of preliminary investigations of these properties have been reported in Refs. $[12,13]$. Here we present results of our studies of the diamond needle-like single crystals aimed to further revealing peculiarities of their photo- and cathodeluminescent characteristics.

\section{Experimental Details}

\subsection{Materials}

The needle-like single crystal diamonds (SCD) were obtained by combination of the CVD and selective oxidation techniques. ${ }^{[10]}$ Home built direct current plasma enhanced CVD allowed creation of the carbon films composed of the pyramid shaped needle-like single crystal diamonds surrounded by a matrix of graphite, nanodiamond, and amorphous carbon. The films were grown on silicon wafer substrate. A selective oxidation at $650^{\circ} \mathrm{C}$ in air atmosphere for about $24 \mathrm{~h}$ was applied to remove the lowordered and graphitic part of CVD film and, thus, to obtain the 


\section{Edited by Foxit PDF Editor \\ Copyright (c) by Foxit Corporation, 2003 - 2010 \\ For Evaluation Only.}

pyramid-shaped diamond crystals in form of powdered material. This powder was used to prepare ethanol-based suspension containing SCD needles. The well-separated diamond crystallites were deposited on the substrates (e.g., on silicon wafers) by subsequent precipitation and drying the drops of the suspension. Detailed description of used methods and facilities are presented elsewhere. ${ }^{[14]}$

\subsection{Cathodoluminescent Measurements}

The cathodoluminescent (CL) investigations were made using specially prepared samples of SCD. Individual diamond crystallites in these samples were disposed on silicon substrates and fixed by local Pt deposition to prevent their uncontrollable moving by electrostatic forces appeared due to charging of dielectric particles by electron beam. The localization and $\mathrm{Pt}$ deposition were made with use of Versa 3D dualbeam (FEI) facility equipped by micromanipulators. The samples with different orientation of the diamond crystallites were prepared for these CL investigations. Scanning electron microscope (SEM) images of some of the samples and simplified scheme of their positioning are presented in Figure 1. It is worth noting that the bottom part of the needle in Figure 1(b) is not fixed on the right side, but it has not got any effects on further investigations. Surface purity of the samples was analyzed by energy-dispersive X-ray spectroscopy (EDS) using INCA EDS analyzer attached to JEOL 7001F scanning electron microscope. Obtained EDS maps did not indicate any detectable impurities on the facets of the SCD samples which were investigated.

The CL measurements were performed using GATAN MONO CL3 attachment to JEOL JSM7001F scanning electron microscope at room temperature $(300 \mathrm{~K})$ and with cooling down to temperature of about $81 \mathrm{~K}$. The electron beam accelerating voltage was varied from 3 to $30 \mathrm{kV}$ and the beam current was changed from $500 \mathrm{pA}$ to $150 \mathrm{nA}$. The electron beam was focused in the area less than $10 \mathrm{~nm}$ in diameter as it was estimated from sample contrast on the SEM images obtained in secondary electrons mode. The diffraction grating with 1200 lines per $\mathrm{mm}$ (sensitivity maximum on $500 \mathrm{~nm}$ wavelength) was used in spectrometer for CL spectra detection. The optimal values of signal to noise ratio and spectral resolution were achieved at $15 \mathrm{kV}$ voltage and $80 \mathrm{nA}$ current of the electron beam. These parameters of the electron beam were used for detection of all CL spectra presented in this work.

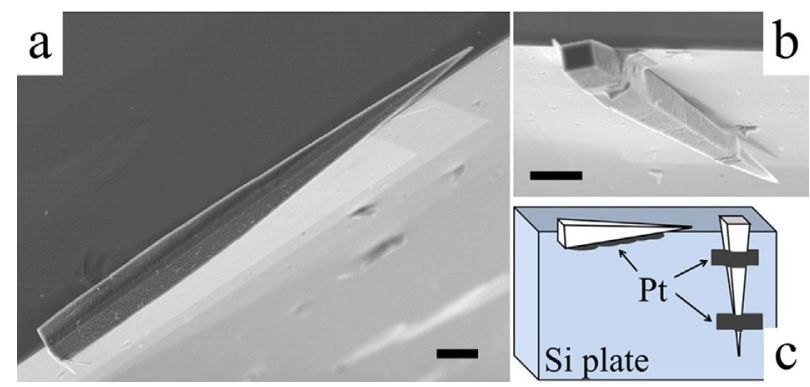

Figure 1. SEM images ( $a$ and $b$ ) and schematic representation (c) of the samples prepared for $\mathrm{CL}$ investigations. Scale bars correspond to $10 \mu \mathrm{m}$.

\subsection{Photoluminescent Measurements}

The needle-like single crystal diamonds were also investigated with photoluminescence (PL) technique. A simplified optical scheme of used facility assembled on optical table is presented in Figure 2. A titanium-sapphire tunable pulsed laser (Chamaleon Ultra from Coherent) with wavelength varied from 690 to $1080 \mathrm{~nm}$ and $80 \mathrm{MHz}$ frequency was used as a source of excitation irradiation. A pulse picker was used to reduce the laser retention rate at $4 \mathrm{MHz}$. Laser irradiation was passed through the second and third harmonic generation block (SHG/THG in Figure 2). To pick out needed wavelength from the generated set of laser radiation color filter was used (excitation filter in Figure 2). Selected laser beam, after passing through the 50/50 ultraviolet beam splitter (BS UV 50/50 in Figure 2) and reflective objective, irradiated the sample. The PL radiation emitted by the sample was collected by the same reflective objective and directed to the imaging camera (if optical image of the sample is required) or to the spectrometer analyzing collected light radiation. An additional optical filter (imaging filter in Figure 2) was installed before the spectrometer to remove intensive scattered radiation of the laser beam or its harmonics. A streak camera (model C5680 from Hamamatsu with slow sweep and an ultimate timing resolution of about $20-30 \mathrm{ps}$ ) situated after the spectrometer also was used for decay time determinations.

The PL investigations were performed under room temperature $(300 \mathrm{~K})$ and with cooling down to $24 \mathrm{~K}$ and to $4 \mathrm{~K}$ temperatures. Wavelength of excitation radiation was varied from 260 to $515 \mathrm{~nm}$. Power of the irradiation beam measured before reflective objective was varied from 50 to $800 \mu \mathrm{W}$. PL spectra were obtained by focusing laser beam at different areas along an individual needle-like diamond crystal.

\section{Results and Discussion}

The advanced experimental techniques used in this study allow obtaining much more details about CL and PL spectra in comparison with our preliminary investigations reported in Refs. $[12,13]$. These details include spectral characteristics of the luminescence, dependence of the spectral lines on temperature and excitation wavelength, their decay time dependence, and phonon vibronic bands characteristics. In the preliminary investigation, we have established that luminescent characteristics of SCD indicate on nitrogen and silicon impurities contaminations. ${ }^{[12,13]}$ While the nitrogen-related color centers are distributed more or less homogeneously in the crystallites,

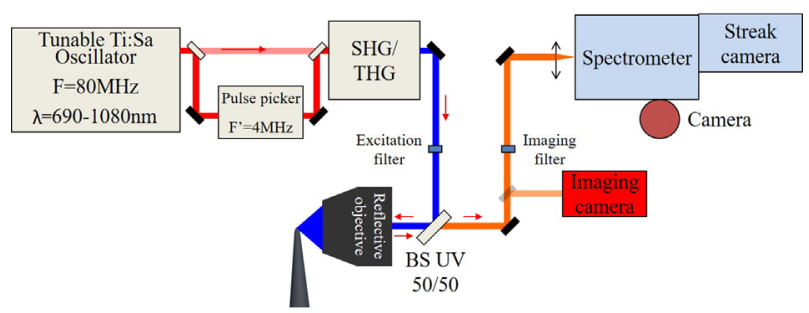

Figure 2. Scheme of the PL setup used for investigation of the diamond needles (see description in text). 


\section{Edited by Foxit PDF Editor \\ Copyright (c) by Foxit Corporation, 2003 - 2010 For Evaluation Only.}

the silicon centers are concentrated predominantly at their apexes. The CL and PL investigations were performed in different areas of SCD (near the apex, in the middle, and near the base). The same luminescence lines in all of these points were observed. In this study, we focused mostly on the analysis of crystallite luminescent properties on the whole. The spectra discussed in this work were observed near the middle of the SCD and are used here as the most representative for the whole crystallite while spectra variations along the needles are discussed in other our works. ${ }^{[12,13]}$

\subsection{Main Luminescence Lines}

The luminescent spectra were obtained in wavelength range of 225-900 $\mathrm{nm}$ with CL technique and (depending on the excitation wavelength) in the range of $260-880 \mathrm{~nm}$ for PL. The spectra in the range of $350-800 \mathrm{~nm}$ are presented in Figures 3 and 4.

Typical CL spectra measured in the middle part of SCD at room temperature and with cooling down to $81 \mathrm{~K}$ are presented in Figure 3. Figure 4 presents the PL spectra obtained at room temperature and at cryogenic temperature $(24 \mathrm{~K})$. At low temperatures, luminescence intensity increases, the width of the lines and, especially, the width of the broad bands related to structural defects in diamond reduces markedly. Overall signalto-noise ratio is increased with temperature decrease that allows appearance of more details in the spectra.

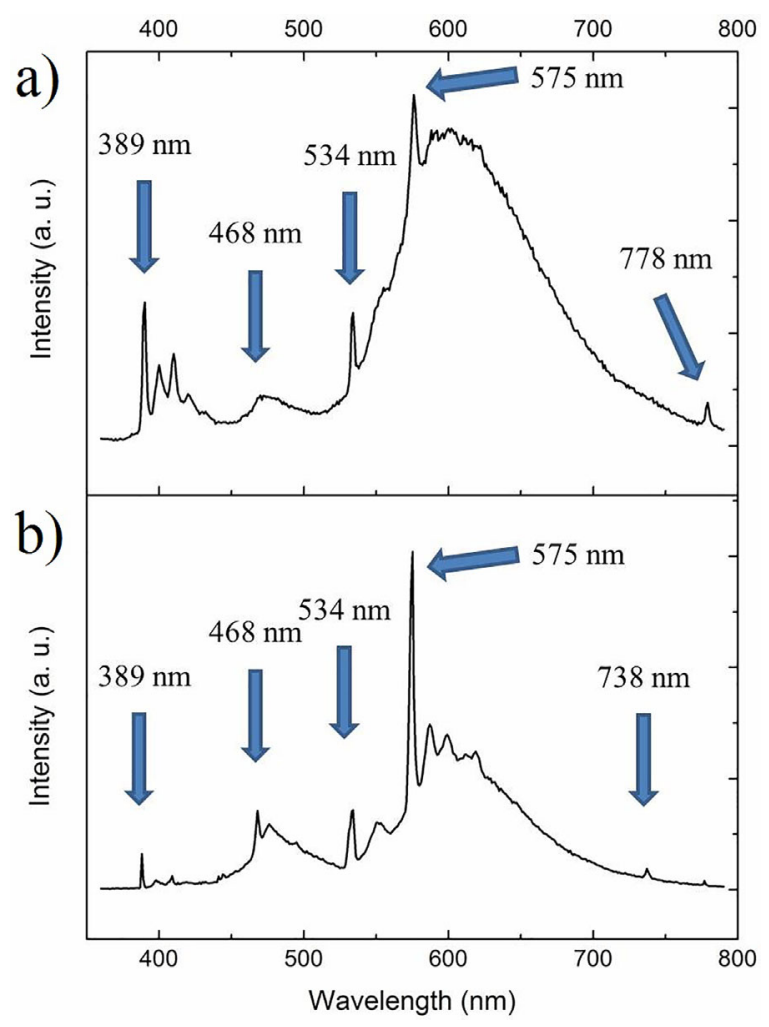

Figure 3. Typical cathodoluminescent spectra obtained at $300 \mathrm{~K}$ (a) and at $81 \mathrm{~K}$ (b). Energy of irradiated electrons is $15 \mathrm{keV}$, beam current is $80 \mathrm{nA}$.

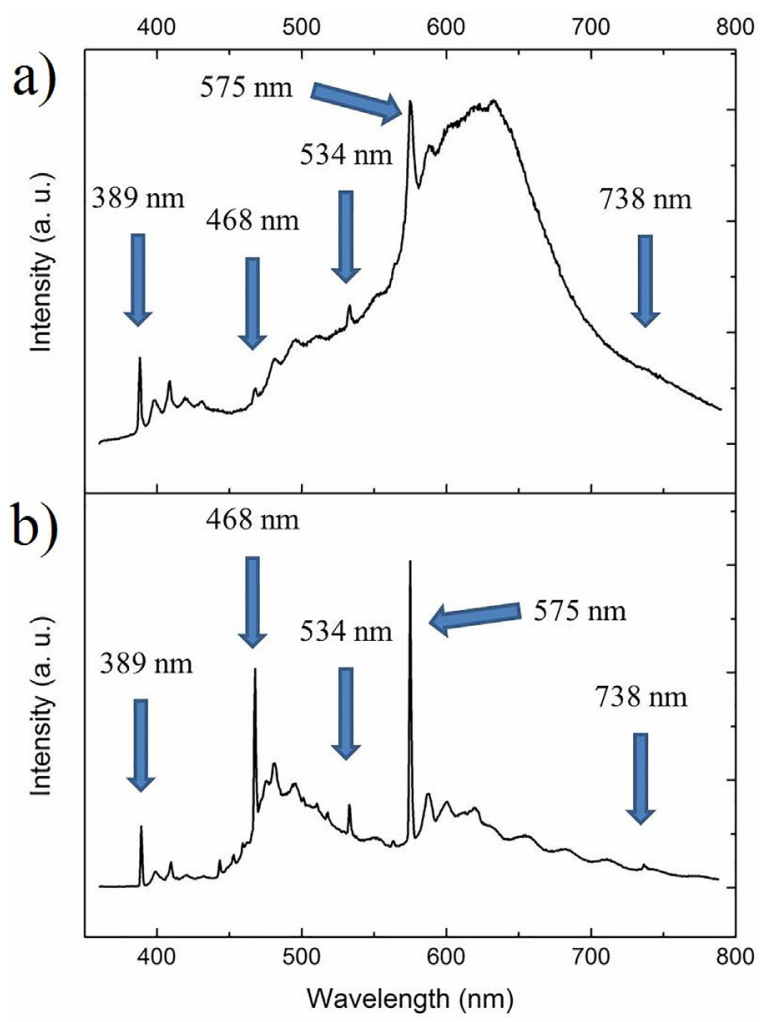

Figure 4. Typical photoluminescent spectra: (a) spectrum measured at sample temperature of $300 \mathrm{~K}$ and with wavelength of excitation radiation of $300 \mathrm{~nm}$; (b) spectrum measured at sample temperature of $24 \mathrm{~K}$ and with wavelength of excitation radiation of $260 \mathrm{~nm}$.

The most intensive sharp lines distinguished in the spectra are centered at $389,442,468,534,563,575$, and $738 \mathrm{~nm}$. The line at $778 \mathrm{~nm}$ presenting in the CL spectra is a second order diffraction of $389 \mathrm{~nm}$ line. The spectral shape of low temperature PL spectrum in $650-730 \mathrm{~nm}$ wavelength range is due to transmission characteristics of the imaging optical filter. Previous investigations ${ }^{[15-17]}$ allow assignment of observed luminescent line at $389 \mathrm{~nm}(3.188 \mathrm{eV})$ to a zero phonon line (ZPL) of nitrogen-related centers in diamond. Intensity of this $389 \mathrm{~nm}$ line was changed significantly during CL signal accumulation for electron beam accelerating voltage above few $\mathrm{kV} .^{[7]}$ It may explain variation of relative intensity of this line in the spectra shown in Figures 3 and 4 . The intensity of similar $389 \mathrm{~nm}$ line have been increased strongly after diamond annealing at temperature from 600 to $800^{\circ} \mathrm{C} .{ }^{[7]}$ Such annealing may occur during oxidation of the CVD diamond film at $650^{\circ} \mathrm{C}$. The nitrogen-related centers proposed for this line may include: defects that contains interstitial nitrogen atoms ${ }^{[18]}$; pseudomolecules $\mathrm{C}-\mathrm{N}$ formed by single substitutional nitrogen atom shifted along the $\langle 111\rangle$ axis ${ }^{[7]}$ or $\mathrm{C}_{2} \mathrm{~N}^{[19]}$; defects containing substitutional nitrogen atom bound to interstitial carbon atoms. ${ }^{[20]}$

The $442 \mathrm{~nm}(2.807 \mathrm{eV})$ line obtained at $24 \mathrm{~K}$ with excitation wavelength of $260 \mathrm{~nm}$ has been assigned also to nitrogen impurities and represents a ZPL observed in the PL spectra. ${ }^{[7]}$ Moreover this line can be observed in low temperature CL 


\section{Edited by Foxit PDF Editor \\ Copyright (c) by Foxit Corporation, 2003 - 2010 \\ For Evaluation Only.}

spectra but it is too weak for detailed analysis. The line is not visible in PL and CL spectra measured at room temperature probably due to large background noise. In accord with previous investigations, the luminescent centers responsible for appearance of $442 \mathrm{~nm}$ line can contain interstitial carbon atoms. ${ }^{[7]}$

The $468 \mathrm{~nm}(2.651 \mathrm{eV})$ line has been observed in high-quality single-crystal CVD diamond films. This line has been assigned to the centers containing nitrogen and interstitial atoms. ${ }^{[7,17]}$ However, in some reports this type of centers was associated also with silicon impurities that appeared in CVD diamond grown on Si substrates. ${ }^{[15,19]}$ Clarification of this contradictory assignments requires high-temperature annealing of diamond in vacuum which was unavailable for us in this work. Obtained spectra demonstrate different shape of $468 \mathrm{~nm}$ line because of vibronic bands which will be discussed in the next section. Intensity of $468 \mathrm{~nm}$ line shows a significant increase in both CL and PL spectra at low temperatures. On the whole $468 \mathrm{~nm}$ line demonstrates greater relative intensity in photo-luminescence in comparison with cathode-luminescence.

The $534 \mathrm{~nm}(2.322 \mathrm{eV})$ line is supposed to be associated with the centers containing nitrogen atoms or nitrogen-vacancy complexes in its structure. ${ }^{[15]}$ Usually the line is observed as a part of ZPL doublet containing also $531 \mathrm{~nm}$ line. But in perfect homoepitaxial CVD diamond films, this line has been observed as a single ZPL line instead. ${ }^{[7]}$ Only single line can be resolved in all spectra obtained in our work (see Figure 3 and 4). This may be considered as an evidence of the high-quality single crystal structure of the needle-like diamonds in consistency with previously reported results. ${ }^{[21]}$ The $534 \mathrm{~nm}$ line is better resolved in CL spectra. It may be due to less intensity of the vibronic bands of $468 \mathrm{~nm}$ line that results to less intensive background.

The $563 \mathrm{~nm}$ line can be detected in PL and sometimes in CL spectra but this line is weaker in comparison with the nearest $575 \mathrm{~nm}$ band. Moreover other lines in this spectral range relate to different defects in diamond. ${ }^{[7]}$

The $575 \mathrm{~nm}(2.156 \mathrm{eV})$ line in the luminescence spectra corresponds to the nitrogen-vacancy centers in uncharged state $\left(\mathrm{NV}^{0}\right){ }^{[22]}$ The properties and structural features of $\mathrm{NV}^{0}$ are investigated in details (see e.g., Ref. [23]). Intensity of vibronic bands associated with $575 \mathrm{~nm}$ line is significantly reduced and the zero phonon line becomes much more pronounced at low temperatures (see Figure 3 and 4).

The decay time of $575 \mathrm{~nm}$ line was investigated with excitation by pulsed laser radiation at $370 \mathrm{~nm}$. Figure 5(a) shows detected
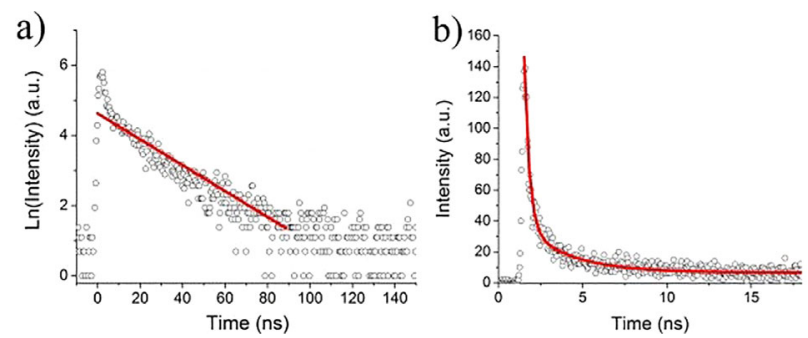

Figure 5. Time dependences of photoluminescent $575 \mathrm{~nm}$ line decay on long (a) and short (b) time scale. Excitation wavelength is $370 \mathrm{~nm}$, beam power is $400 \mu \mathrm{W}$. Red lines correspond to exponential decay adjustments in semi-logarithmic (a) and linear scale (b). time dependence of PL intensity after pulse irradiation on long time scale of $200 \mathrm{~ns}$. The decay time of neutral nitrogen-vacancy color centers luminescence calculated from this data is about $28 \pm 1 \mathrm{~ns}$. This value is in agreement with previously reported values of about 29-32 ns for the decay time of $575 \mathrm{~nm}$ line. ${ }^{[7,24]}$ However, our measurements indicate that in short time scale $(20 \mathrm{~ns})$ a fast decay of luminescence intensity with characteristics time of $2.8 \pm 0.3 \mathrm{~ns}$ also occurs (see Figure 5(b)). Similar characteristics decay time has been previously observed in diamonds. ${ }^{[25]}$

The $738 \mathrm{~nm}(1.681 \mathrm{eV})$ line has been assigned to another wellknown color center in diamond related to silicon impurities (so called silicon or silicon-vacancy (SiV) color center). ${ }^{[26]}$ Investigations of this center in recent year have been mostly stimulated by its potential usage in quantum optical devices. ${ }^{[27]}$ Atomic structure of SiV center has been supposed as interstitial silicon atom between two substitutional vacancies. ${ }^{[28]}$ The center is known to exist in neutral and negative charge states. The line at $738 \mathrm{~nm}$ corresponds to its negatively charged state. Only single ZPL line may be resolved in CL and PL spectra obtained in our work.

The intensities of the lines at 575 and $738 \mathrm{~nm}$ were found to be dependent on wavelength of excitation radiation. The dependencies obtained for the ratios of PL line amplitude to base signal are shown in Figure 6. For the $738 \mathrm{~nm}$ line, the relative intensity increases linearly with the laser wavelength, suggesting that excitation of these centers with longer wavelength (around $500 \mathrm{~nm}$ ) is preferable. ${ }^{[7]}$ The linear intensity dependence is in consistency with PL excitation spectra reported in previous studies. ${ }^{[29]}$ In contrast, the $575 \mathrm{~nm}$ line shows a non-monotonic (parabolic) variation of excitation efficiency, which increases of a factor three from 400 to $500 \mathrm{~nm}$. Similar strong dependencies of $575 \mathrm{~nm}$ line on excitation wavelength has been explained by

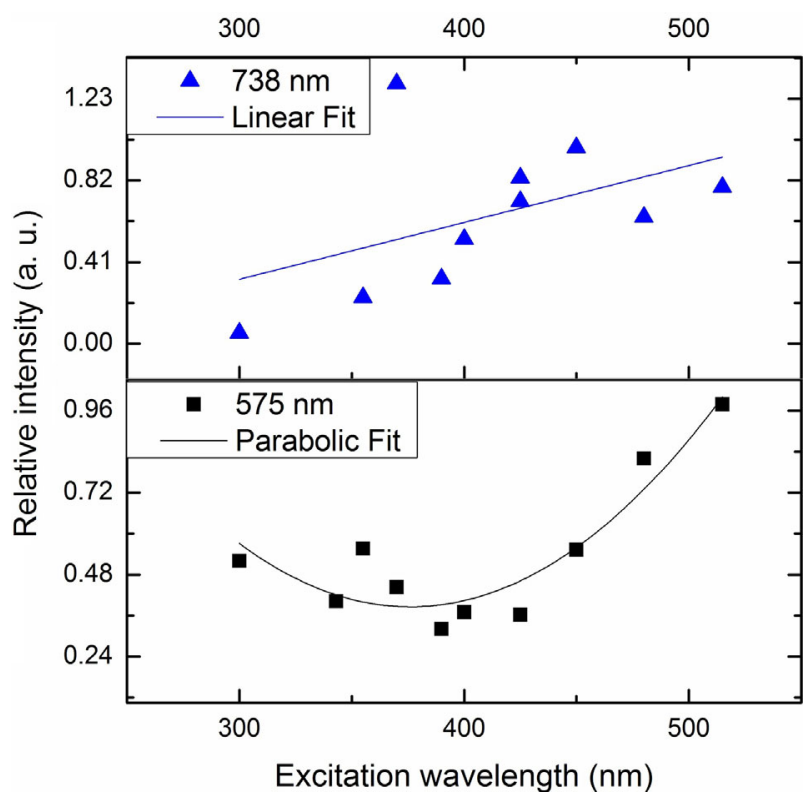

Figure 6. Photoluminescent intensity dependence for 575 and $738 \mathrm{~nm}$ on excitation wavelength measured for the area located near the apex of diamond needle. 


\section{Edited by Foxit PDF Editor \\ Copyright (c) by Foxit Corporation, 2003 - 2010 \\ For Evaluation Only.}

increasing of absorption efficiency in the range near $415 \mathrm{~nm}$ $(3 \mathrm{eV}) \cdot{ }^{[30]}$

\subsection{Vibronic Bands}

Besides of the ZPL lines the luminescent spectra of SCD contains pronounced phonon replications (or vibronic) bands for most of the color centers. Figure 7 presents dependencies of the spectra in vicinity of the ZPL lines with phonon replications on the phonon energy. The phonon energies were calculated from wavelength in luminescent spectra (similar to that shown in Figures 3 and 4) considering phonon energy equal to zero for the wavelength of corresponding ZPL lines. The spectra presented in Figure 7 were normalized on intensity of corresponding ZPL lines and shifted in vertical direction for clarity.

The most of the vibronic bands detected in spectra shown in Figure 7 are in well consistency with previous investigations. The observed vibronic bands of $389 \mathrm{~nm}$ ZPL line are appeared in PL and CL spectra at $80 \pm 6,159 \pm 6$, and $236 \pm 6 \mathrm{meV}$ that corresponds to electron transitions with local vibrational modes associated with $75 \mathrm{meV}$ transverse acoustic (TA) phonon ${ }^{[31]}$ and $165 \mathrm{meV}$ longitudinal optical (LO) phonon. ${ }^{[7]}$ Interaction of $389 \mathrm{~nm}$ transition with both of these phonons can explain appearance of $236 \pm 6 \mathrm{meV}$ feature in the spectra. ${ }^{\text {[7] }}$ The observed vibronic bands of $442 \mathrm{~nm}$ line (Figure $7(\mathrm{~b})$ ) correspond to phonon energies of $58 \pm 4$ and $96 \pm 4 \mathrm{meV}$.

Four phonon bands can be resolved for $468 \mathrm{~nm}$ line in its side band (Figure 7(c)). Predominant interaction with $76 \pm 4 \mathrm{meV}$ phonon (similar to that described in Ref. [7]) corresponds to the bands observed at $148 \pm 5$ and $220 \pm 4 \mathrm{meV}$ for two- and threephonon processes, respectively. However, the line corresponding
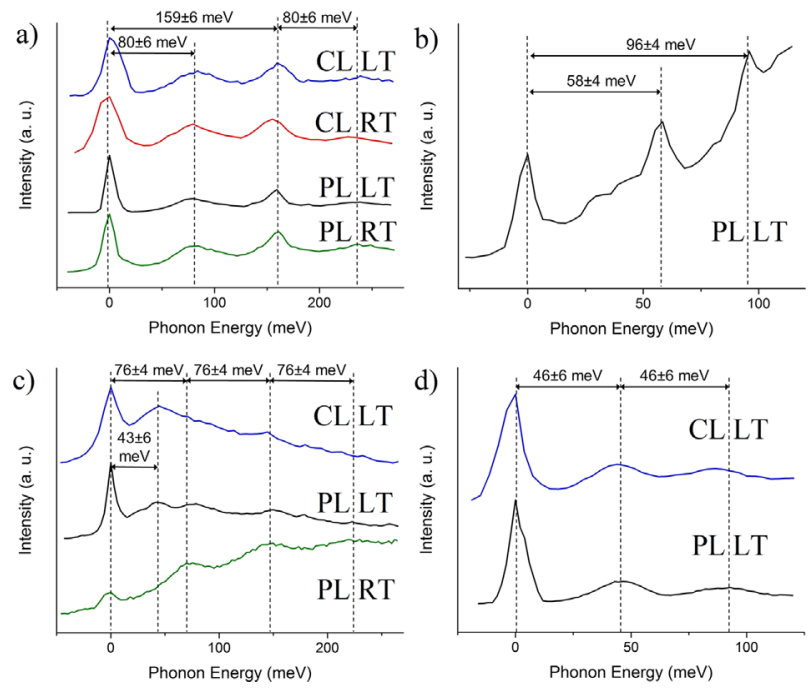

Figure 7. Vibronic bands for $389 \mathrm{~nm}$ (a), $442 \mathrm{~nm}$ (b), $468 \mathrm{~nm}$ (c), and $575 \mathrm{~nm}$ (d) centers. Blue lines (CL LT) correspond to cathodoluminescent spectra measured at $81 \mathrm{~K}$, red lines $(C L R T)$ correspond to cathodoluminescent spectra measured at room temperature, black lines (PL LT) correspond to photoluminescence spectra measured at low temperatures (24 K for (a) and (b), $4 \mathrm{~K}$ for (c) and (d)), green lines (PL RT) correspond to photoluminescence spectra measured at room temperature.

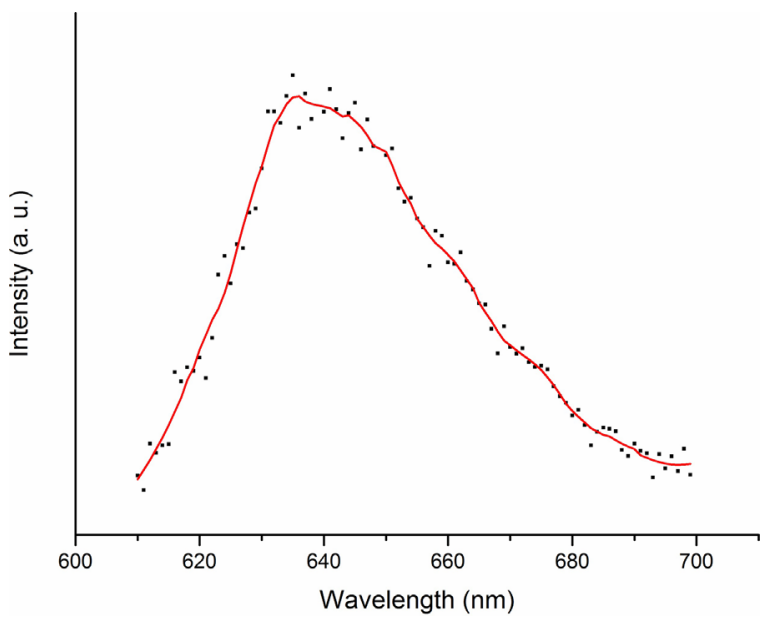

Figure 8. Spectral dependence obtained by subtraction of normalized CL spectrum from PL spectrum (shown in Figure 3(a) and 4(a), respectively) indicated by black dots. The red line corresponds to smoothed data.

to phonon with $43 \pm 6 \mathrm{meV}$ was also detected in some spectra for this optical center. This line can be either additional vibrational band of 442 or $468 \mathrm{~nm}$ lines or independent luminescence line with wavelength about $477 \mathrm{~nm}$. For $534 \mathrm{~nm}$ line one vibrational band can be resolved. It corresponds to phonon with energy of $79 \pm 8 \mathrm{meV}$ that in consistence with previous researches. ${ }^{[7]}$ The phonon interactions with energies of $46 \pm 6$ and $90 \pm 6 \mathrm{meV}$ that corresponds to two-phonon process with $46 \pm 6 \mathrm{meV}$ can be resolved in spectral range of $575 \mathrm{~nm}$ line (Figure 7 (d)) similar to previous reports. ${ }^{[32]}$

Some difference between CL and PL spectra (Figures 3(a) and 4(a)) can be seen for vibronic bands of $575 \mathrm{~nm}$ ZPL line. The PL spectrum looks wider and more intensive around $640 \mathrm{~nm}$ wavelength. Subtraction of normalized CL spectrum from PL spectrum (see Figure 8) produces dependence with shape similar to spectrum of negatively charged nitrogen-vacancy center in diamond with $637 \mathrm{~nm}$ wavelength. This is in well agreement with previous reports indicating that negatively charged nitrogen-vacancy center is not detectable by CL technique. ${ }^{[7]}$

\section{Conclusion}

The 389, 442, 468, 534, 563, 575, and $738 \mathrm{~nm}$ luminescent lines of the color centers in needle-like single crystal diamond were observed. Single zero phonon line at $534 \mathrm{~nm}$, strong luminescence at $468 \mathrm{~nm}$, and the vibration modes observed in the spectra demonstrate high structural quality of manufactured needle-like single diamond crystals. The presence of 389 and $468 \mathrm{~nm}$ luminescent lines indicates that these structures also contain interstitial and/or substitutional atoms. The luminescent lines at 389, 468, 534, and $575 \mathrm{~nm}$ demonstrate a nitrogen atoms content. The $738 \mathrm{~nm}$ line is an evidence of silicon atom impurities presence. Pronounced vibronic bands in the luminescent spectra of nitrogen-vacancy centers correspond to interactions with: $80 \pm 6$ and $159 \pm 6 \mathrm{meV}$ phonons for $389 \mathrm{~nm}$ line, $58 \pm 4$ and $96 \pm 4 \mathrm{meV}$ phonons for $442 \mathrm{~nm}$ line; 


\section{Edited by Foxit PDF Editor Copyright (c) by Foxit Corporation, 2003 - 2010 For Evaluation Only.}

$76 \pm 4 \mathrm{meV}$ phonons for $468 \mathrm{~nm}$ line; $46 \pm 6 \mathrm{meV}$ phonons for $575 \mathrm{~nm}$ line.

\section{Acknowledgments}

This work was supported by the French "I'Agence Nationale de la Recherche (ANR)," through the program "Investissements d'Avenir" (ANR-10-LABX-09-01), LabEx EMC3, ASAP project. SAM and ANO are grateful for support from Russian Science Foundation (grant no. 15 02-30041).

\section{Conflict of Interest}

The authors declare no conflict of interest.

\section{Keywords}

cathodoluminescence, chemical vapor deposition, diamond, needle-like crystals, photoluminescence

[1] B. Nöhammer, J. Hoszowska, A. K. Freund, C. David, J. Synchrotron Radiat. 2003, 10, 168.

[2] K. F. Chong, K. P. Loh, K. Ang, Y. P. Ting, Analyst 2008, 133, 739.

[3] M. Akaishi, H. Kanda, S. Yamaoka, J. Cryst. Growth 1990, 104, 578.

[4] B. V. Spitsyn, L. L. Bouilov, B. V. Derjaguin, J. Cryst. Growth 1981, 52, 219.

[5] H. K. Tönshoff, H. Hillmann-Apmann, J. Asche, Diam. Relat. Mater. 2002, 11, 736.

[6] J. Isberg, A. Lindblom, A. Tajani, D. Twitchen, Phys. Status Solidi A 2005, 202, 2194.

[7] A. M. Zaitsev, Optical Properties of Diamond, Springer-Verlag, Berlin, Heidelberg 2001.

[8] T. M. Babinec, B. J. M. Hausmann, M. Khan, Y. Zhang, J. R. Maze, P. R. Hemmer, M. Loncar, Nature Nanotechnol. 2010, 5, 195.

[9] V. M. Acosta, E. Bauch, M. P. Ledbetter, C. Santori, K.-M. C. Fu, P. E. Barclay, R. G. Beausoleil, H. Linget, J. F. Roch, F. Treussart,
S. Chemerisov, W. Gawlik, D. Budker, Phys. Rev. B 2009, 80, 115202.

[10] A. N. Obraztsov, P. G. Kopylov, A. L. Chuvilin, N. V. Savenko, Diam. Relat. Mater. 2009, 18, 1289.

[11] A. A. Zolotukhin, M. A. Dolganov, A. M. Alekseev, A. N. Obraztsov, Diam. Relat. Mater. 2014, 42, 15.

[12] F. T. Tuyakova, E. A. Obraztsova, E. V. Korostylev, D. V. Klinov, K. A. Prusakov, A. A. Alekseev, R. R. Ismagilov, A. N. Obraztsov, J. Lumin. 2016, 179, 539.

[13] A. M. Alekseev, F. T. Tuyakova, E. A. Obraztsova, E. V. Korostylev, D. V. Klinov, K. A. Prusakov, S. A. Malykhin, R. R. Ismagilov, A. N. Obraztsov, Phys. Solid State 2016, 58, 2307.

[14] P. G. Kopylov, A. N. Obraztsov, P. V. Shvets, Crystallogr. Rep. 2010, 55, 710.

[15] J. Ruan, W. J. Choyke, W. D. Partlow, J. Appl. Phys. 1991, 69, 6632.

[16] A. T. Collins, Diam. Relat. Mater. 1992, 1, 457.

[17] K. lakoubovskii, G. J. Adriaenssens, Phys. Rev. B 2000, 61, 10174.

[18] A. T. Collins, M. Kamo, Y. Sato, J. Phys. D: Appl. Phys. 1989, 22, 1402.

[19] B. Dischler, W. Rothemund, K. Maier, C. Wild, H. Biebl, P. Koidl, Diam. Relat. Mater. 1994, 3, 825.

[20] A. T. Collins, P. J. Woad, G. S. Woods, H. Kanda, Diam. Relat. Mater. 1993, 2, 136.

[21] A. S. Orekhov, F. T. Tuyakova, E. A. Obraztsova, A. B. Loginov, A. L. Chuvilin, A. N. Obraztsov, Nanotechnology 2016, 27, 455707.

[22] T. Gaebel, M. Domhan, C. Wittmann, I. Popa, F. Jelezko, J. Rabeau, A. Greentree, S. Prawer, E. Trajkov, P. R. Hemmer, J. Wrachtrup, Appl. Phys. B 2005, 82, 243.

[23] M. W. Doherty, N. B. Manson, P. Delaney, F. Jelezko, J. Wrachtrup, L. C. L. Hollenberg, Phys. Rep. 2013, 528, 1.

[24] E. Pereira, L. Pereira, R. Raue, Diam. Relat. Mater. 1992, 1, 901.

[25] H. Hanzawa, Y. Nisida, T. Kato, Diam. Relat. Mater. 1997, 6, 1595.

[26] C. Hepp, T. Müller, V. Waselowski, J. N. Becker, B. Pingault, H. Sternschulte, D. Steinmüller-Nethl, A. Gali, J. R. Maze, M. Atatüre, C. Becher, Phys. Rev. Lett. 2014, 112, 036405.

[27] E. Neu, D. Steinmetz, J. Riedrich-Möller, S. Gsell, M. Fischer, M. Schreck, C. Becher, New J. Phys. 2011, 13, 025012.

[28] L. J. Rogers, K. D. Jahnke, M. W. Doherty, A. Dietrich, L. P. McGuinness, C. Müller, T. Teraji, H. Sumiya, J. Isoya, N. B. Manson, F. Jelezko, Phys. Rev. B 2014, 89, 235101.

[29] K. lakoubovskii, G. Adriaenssens, M. Nesládek, L. Stals, Diam. Relat. Mater. 1999, 8, 717.

[30] J. Walker, Rep. Prog. Phys. 1979, 42, 1605.

[31] A. M. Zaitsev, Phys. Rev. B 2000, 61, 12909.

[32] B. Dischler, Handbook of Spectral Lines in Diamond, Springer-Verlag, Berlin, Heidelberg 2012. 